INJE-TP-95-2

\title{
Blueshift of tachyon in the charged 2D black hole
}

\author{
H.W. Lee and Y. S. Myung \\ Department of Physics, Inje University, Kimhae 621-749, Korea \\ Jin Young Kim \\ Division of Basic Science, Dongseo University, Pusan 616-010, Korea
}

\begin{abstract}
We study the propagation of string fields (metric $G_{\mu \nu}$, Mawxell gauge potential $A_{\mu}$, dilaton $\Phi$, and tachyon $T$ ) in a two-dimensional (2D) charged black hole. It is shown that the tachyon is a propagating field both inside and outside the black hole. This becomes infinitely blueshifted at the inner horizon. We confirm that the inner horizon is unstable, whereas the outer horizon is stable.
\end{abstract}

PACS number(s) : 04.70.Bw, 11.25 Db, 11.55.Ds 
Lower dimensional theories of gravity provide a simplified context in which to study black hole physics [1]. The non-triviality of these models arises from the non-minimal coupling of the dilaton to the scalar curvature. A dilaton potential of the type produced by the string loop corrections may induce multiple horizons [2]. For example, the 2D charged black hole from heterotic string theories has shown this feature. This has many analogies with the Reissner-Nordström black hole in $4 \mathrm{D}$ general relativity. In addition to the event (outer) horizon $\left(r_{+}\right)$, there exist the Cauchy (inner) horizon $\left(r_{-}\right)$in both the 2D charged and Reissner-Nordström black hole.

Penrose [3] has first pointed out that the Cauchy horizon of the Kerr-Newman black hole in $4 \mathrm{D}$ gravity is unstable due to the infinite blueshift of the infalling radiation. Here the large blueshift of infalling matter means a general divergence of the field energy density as the evolution approaches the Cauchy horizon. McNarama [4] has demonstrated that a test scalar field evolved to have unbounded enegy density on $r_{-}$. The literatures in [5-8] considered the stability problem of the Cauchy horizon within the Reissner-Nordström geometry. These indicated that the Cauchy horizon is unstable to the external perturbations. On the other hand, Poisson and Israel [9] showed that the mass parameter near $r_{-}$becomes unbounded only when both the infalling and outgoing radiations are present. This is called the mass inflation. Ori [10] confirmed this mass inflation within a simplified model. Recently, Husain [11] showed that the mass inflation also occurs in the 3D space-time. More recently, Chan and Mann [12] investigated the same problem in the 2D charged dilaton gravity. In this case the stress-energy tensor for matter is taken as a null fluid.

This paper is concentrated on the study of the propagation of string fields (metric $G_{\mu \nu}$, Mawxell gauge potential $A_{\mu}$, dilaton $\Phi$, and tachyon $T$ ) in the 2D charged black hole. We will show that the tachyon is the only propagating field in the background of double horizons. Following Ref.[6], we use the tachyon to investigate inner and outer horizons instead of a null fluid matter. Furthermore, the region outside the black hole is also studied when the charge of the black hole vanishes $(Q=0)$.

From the conformal invariance of the heterotic 2D string theories, one can derive the 
$\beta$-function equations $[2,13,14]$

$$
\begin{gathered}
R_{\mu \nu}+2 \nabla_{\mu} \nabla_{\nu} \Phi-F_{\mu \rho} F_{\nu}{ }^{\rho}-\frac{1}{2} \nabla_{\mu} T \nabla_{\nu} T=0 \\
\nabla^{2} \Phi-2(\nabla \Phi)^{2}+\frac{1}{2} \alpha^{2}+\frac{1}{4} F^{2}+\frac{1}{2} T^{2}=0 \\
\nabla_{\mu} F^{\mu \nu}-2\left(\nabla_{\mu} \Phi\right) F^{\mu \nu}=0 \\
\nabla^{2} T-2 \nabla_{\mu} \Phi \nabla^{\mu} T+2 T=0
\end{gathered}
$$

where $F_{\mu \nu}=\partial_{[\mu} A_{\nu]}$ is the Maxwell field. The above equations are also derived from the requirement that the fields must be an extremum of the low-energy string action [14]

$$
S_{l-e}=\int d^{2} x \sqrt{-G} e^{-2 \Phi}\left\{R+4(\nabla \Phi)^{2}+\alpha^{2}-\frac{1}{2} F^{2}-\frac{1}{2}(\nabla T)^{2}+T^{2}\right\} .
$$

For an example, we consider the variation of $S_{l-e}$ with respect to the metric $G_{\mu \nu}$. This leads to $T_{\mu \nu}=0$ with the stress-energy tensor

$$
\begin{gathered}
T_{\mu \nu}=T_{\mu \nu}^{\Phi}+T_{\mu \nu}^{t}+T_{\mu \nu}^{F}=-2 \nabla_{\mu} \nabla_{\nu} \Phi+2 G_{\mu \nu}\left(\nabla^{2} \Phi-(\nabla \Phi)^{2}+2\right) \\
+\frac{1}{2} \nabla_{\mu} T \nabla_{\nu} T+\frac{1}{4} G_{\mu \nu}\left(2 T^{2}-(\nabla T)^{2}\right)+F_{\mu \rho} F_{\nu} \rho-\frac{1}{4} G_{\mu \nu} F^{2} .
\end{gathered}
$$

For convenience, let us take the following transformation

$$
-2 \Phi \rightarrow \Phi, \quad T \rightarrow \sqrt{2} T, \quad-R \rightarrow R .
$$

Then the equations of motion are given by

$$
\begin{aligned}
& R_{\mu \nu}+\nabla_{\mu} \nabla_{\nu} \Phi+F_{\mu \rho} F_{\nu}{ }^{\rho}+\nabla_{\mu} T \nabla_{\nu} T=0, \\
& (\nabla \Phi)^{2}+\nabla^{2} \Phi-\frac{1}{2} F^{2}-2 T^{2}-8=0, \\
& \nabla_{\mu} F^{\mu \nu}+\left(\nabla_{\mu} \Phi\right) F^{\mu \nu}=0, \\
& \nabla^{2} T+\nabla \Phi \nabla T+2 T=0,
\end{aligned}
$$


where we set $\alpha^{2}=8$. The equation (9) comes from $T_{\mu}{ }^{\mu}=0$ with the substitution (7). For a later purpose, we have the stress-energy tensor for tachyon (after substitution of (7))

$$
T_{\mu \nu}^{t}=\nabla_{\mu} T \nabla_{\nu} T+\frac{1}{2} G_{\mu \nu}\left(2 T^{2}-(\nabla T)^{2}\right) .
$$

The charged black hole solution to the above equations is given by setting the tachyon $(T)$ to zero $[2]$

$$
\bar{\Phi}=2 \sqrt{2} r, \quad \bar{F}_{t r}=Q e^{-2 \sqrt{2} r}, \quad \bar{T}=0, \quad \bar{G}_{\mu \nu}=\left(\begin{array}{cc}
-f & 0 \\
0 & f^{-1}
\end{array}\right)
$$

with

$$
f=1-e^{-2 \sqrt{2} r}+\frac{Q^{2}}{8} e^{-4 \sqrt{2} r}
$$

Introducing the new coordinate $y=\exp (2 \sqrt{2} r), f=\frac{1}{y^{2}}\left(y^{2}-y+\frac{Q^{2}}{8}\right)$. From $f=0$, the double horizons $\left(r_{ \pm}\right)$are given by

$$
r_{ \pm}=\frac{1}{2 \sqrt{2}} \log y_{ \pm}
$$

with

$$
y_{ \pm}=\frac{1 \pm \sqrt{1-\frac{Q^{2}}{2}}}{2}
$$

Here $r_{+}\left(r_{-}\right)$correspond to the outer (inner) horizons. In the case of $Q=1$, we have $r_{+}=-0.056$ and $r_{-}=-0.679$.

To study the propagation of string fields, we introduce small perturbation fields around the background solution as [15]

$$
\begin{aligned}
& F_{t r}=\bar{F}_{t r}+\mathcal{F}_{t r}=\bar{F}_{t r}\left[1-\frac{\mathcal{F}(r, t)}{Q}\right], \\
& \Phi=\bar{\Phi}+\phi(r, t), \\
& G_{\mu \nu}=\bar{G}_{\mu \nu}+h_{\mu \nu}=\bar{G}_{\mu \nu}[1-h(r, t)], \\
& T=\bar{T}+\tilde{t} \equiv \exp \left(-\frac{\bar{\Phi}}{2}\right)[0+t(r, t)] .
\end{aligned}
$$


where we choose the metric perturbation $\left(h_{\mu \nu}\right)$ in such a way that the background symmetry should be restored at the perturbation level. This is an important point in studying all black holes $[7,15]$. One linearizes (8)-(11) in order to obtain the equations governing the perturbation as

$$
\begin{gathered}
\delta R_{\mu \nu}(h)+\bar{\nabla}_{\mu} \bar{\nabla}_{\nu} \phi-\delta \Gamma_{\mu \nu}^{\rho}(h) \bar{\nabla}_{\rho} \bar{\Phi}+2 \bar{F}_{\mu \rho} \mathcal{F}_{\nu}{ }^{\rho}-\bar{F}_{\mu \rho} \bar{F}_{\nu \alpha} h^{\rho \alpha}=0 \\
-h^{\mu \nu} \bar{\nabla}_{\mu} \bar{\nabla}_{\nu} \bar{\Phi}-\bar{G}^{\mu \nu} \delta \Gamma_{\mu \nu}^{\rho}(h) \partial_{\rho} \bar{\Phi}+\bar{\nabla}^{2} \phi-h^{\mu \nu} \partial_{\mu} \bar{\Phi} \partial_{\nu} \bar{\Phi}+2 \bar{G}^{\mu \nu} \partial_{\mu} \bar{\Phi} \partial_{\nu} \phi \\
-\bar{F}_{\mu \nu} \mathcal{F}^{\mu \nu}+\bar{F}_{\mu \nu} \bar{F}_{\rho}{ }^{\nu} h^{\mu \rho}=0 \\
\left(\bar{\nabla}_{\mu}+\partial_{\mu} \bar{\Phi}\right)\left(\mathcal{F}^{\mu \nu}-\bar{F}_{\alpha}{ }^{\nu} h^{\alpha \mu}-\bar{F}^{\mu}{ }_{\beta} h^{\beta \nu}\right)+\bar{F}^{\mu \nu}\left(\delta \Gamma_{\sigma \mu}^{\sigma}(h)+\left(\partial_{\mu} \phi\right)\right)=0 \\
\bar{\nabla}^{2} \tilde{t}+\bar{\nabla}_{\mu} \bar{\Phi} \bar{\nabla}^{\mu} \tilde{t}+2 \tilde{t}=0
\end{gathered}
$$

where

$$
\begin{aligned}
& \delta R_{\mu \nu}(h)=\frac{1}{2} \bar{\nabla}_{\mu} \bar{\nabla}_{\nu} h_{\rho}^{\rho}+\frac{1}{2} \bar{\nabla}^{\rho} \bar{\nabla}_{\rho} h_{\mu \nu}-\frac{1}{2} \bar{\nabla}^{\rho} \bar{\nabla}_{\nu} h_{\rho \mu}-\frac{1}{2} \bar{\nabla}^{\rho} \bar{\nabla}_{\mu} h_{\nu \rho}, \\
& \delta \Gamma_{\mu \nu}^{\rho}(h)=\frac{1}{2} \bar{G}^{\rho \sigma}\left(\bar{\nabla}_{\nu} h_{\mu \sigma}+\bar{\nabla}_{\mu} h_{\nu \sigma}-\bar{\nabla}_{\sigma} h_{\mu \nu}\right) .
\end{aligned}
$$

From (23) one can express $\mathcal{F}$ in terms of $\phi$ and $h$ as

$$
\mathcal{F}=-Q(\phi+h) .
$$

This means that $\mathcal{F}$ is no longer an independent mode. Also from the diagonal element of (21), we have

$$
\begin{aligned}
& \bar{\nabla}^{2} h-2 \bar{\nabla}_{t}^{2} \phi-2 \sqrt{2} \bar{G}^{r r} \partial_{r} h+\bar{F}^{2}(h+2 \phi)=0, \\
& \bar{\nabla}^{2} h-2 \bar{\nabla}_{r}^{2} \phi+2 \sqrt{2} \bar{G}^{r r} \partial_{r} h+\bar{F}^{2}(h+2 \phi)=0 .
\end{aligned}
$$

Adding the above two equations leads to

$$
\bar{\nabla}^{2}(h-\phi)+\bar{F}^{2}(h+2 \phi)=0 .
$$


And the off-diagonal element of (21) takes the form

$$
\partial_{t}\left\{\left(\partial_{r}-\Gamma_{t r}^{t}\right) \phi+\sqrt{2} h\right\}=0
$$

Also the dilaton equation (22) together with (27) leads to

$$
\bar{\nabla}^{2} \phi+4 \sqrt{2} f \partial_{r} \phi+2 \sqrt{2}\left(\partial_{r} f+2 \sqrt{2} f\right) h+\bar{F}^{2} \phi=0
$$

From (31), the relation between $\phi$ and $h$ is given by

$$
\sqrt{2} h=-\partial_{r} \phi+\frac{1}{2} \frac{\partial_{r} f}{f} \phi+U(r)
$$

Here $U(r)$ is the residual gauge degrees of freedom and thus we set $U(r)=0$ for simplicity. Substituting (33) into (32), we have

$$
\bar{\nabla}^{2} \phi+2 \sqrt{2} \partial_{r} f(h+\phi)+\bar{F}^{2} \phi=0 .
$$

Calculating $(30)+2 \times(34)$, one finds the other equation

$$
\bar{\nabla}^{2}(h+\phi)+4 \sqrt{2} \partial_{r} f(h+\phi)+\bar{F}^{2}(h+4 \phi)=0 .
$$

In the beginning we started with two fields $(h, \phi)$. However, from (30) and (35) we have four modes $(h-\phi, h+\phi, h+2 \phi, h+4 \phi)$. When $Q \neq 0$, it is not easy to find out the solutions which satisfy both (30) and (35). Instead, we first check whether the graviton $(h)$ and dilaton $(\phi)$ are physically propagating modes in the 2D charged black hole blackground. We consider the conventional counting of degrees of freedom. The number of degrees of freedom for the gravitational field $\left(h_{\mu \nu}\right)$ in $d$-dimensions is $(1 / 2) d(d-3)$. For a $d=4$ Schwarzschild black hole, we obtain two degrees of freedom. These correspond to the Regge-Wheeler mode for odd-parity perturbation and Zerilli mode for even-parity perturbation. We have -1 for $d=2$. This means that in two dimensions the contribution of graviton is equal and opposite to that of a spinless particle (dilaton). In the $2 \mathrm{D}$ dilaton black hole $(Q=0)$, two gravitondilaton modes $(h-\phi, h+\phi)$ are thus trivial gauge artifacts [16,17]. In addition, a Maxwell field is introduced in the charged 2D black hole. The Maxwell field has $d-2$ physical degrees 
of freedom. For $d=2$, Maxwell field has no physical degrees of freedom. We confirm this from (27). We thus insist that graviton-dilaton, and Maxwell modes become gauge artefacts in the charged 2D black hole. Since these are not physically propagating modes, it is not necessary to consider (30) and (35).

One remaining equation that describes a physically propagating mode is just the tachyon equation (24), which can be rewritten as

$$
f^{2} t^{\prime \prime}+f f^{\prime} t^{\prime}-\left[\sqrt{2} f f^{\prime}-2 f(1-f)\right] t-\frac{\partial^{2}}{\partial t^{2}} t=0
$$

where the prime (I) denotes the derivative with respect to $r$. In order to study the tachyonic propagation, we transform (36) into the form of Schrödinger equation. Introducing the coordinate transformation

$$
r \rightarrow r^{*} \equiv g(r)
$$

then (36) can be rewritten as

$$
f^{2} g^{\prime 2} \frac{\partial^{2}}{\partial r^{* 2}} t+f\left\{f g^{\prime \prime}+f^{\prime} g^{\prime}\right\} \frac{\partial}{\partial r^{*}} t-\left[\sqrt{2} f f^{\prime}-2 f(1-f)\right] t-\frac{\partial^{2}}{\partial t^{2}} t=0 .
$$

Requiring that the coefficient of the linear derivative vanish, one finds the relation

$$
g^{\prime}=\frac{1}{f}
$$

From this relation one can determine the explicit form of $r^{*}$,

$$
r^{*}=g(r)=\frac{1}{\kappa_{+}} \log \left|e^{2 \sqrt{2} r}-e^{2 \sqrt{2} r_{+}}\right|-\frac{1}{\kappa_{-}} \log \left|e^{2 \sqrt{2} r}-e^{2 \sqrt{2} r_{-}}\right|,
$$

with the surface gravity at $r_{ \pm}$defined as

$$
\kappa_{ \pm}=\frac{2 \sqrt{2}\left(y_{+}-y_{-}\right)}{y_{ \pm}}
$$

Assuming $t\left(r^{*}, t\right) \sim t_{\omega}\left(r^{*}\right) e^{-i \omega t}$, one can cast (37) into the one-dimensional Schrödinger equation

$$
\left\{\frac{\partial^{2}}{\partial r^{* 2}}+\omega^{2}-V_{T}(r)\right\} t_{\omega}=0
$$


where the effective potential $V_{T}(r)$ is given by

$$
V_{T}(r)=f\left(\sqrt{2} f^{\prime}-2(1-f)\right)
$$

As is shown in Fig.1, $V_{T}(r)$ is a double-humped barrier well $\left(V_{T}^{I N}\right)$ between the Cauchy horizon and event horizon, while it is just a potential barrier $\left(V_{T}^{\text {OUT }}\right)$ outside the event horizon.

First we consider the region inside the black bole. It is very important to note that inside the black hole the radial coordinate $\left(r\right.$ or $\left.r^{*}\right)$ is timelike, whereas the time $(t)$ is spacelike. Hence to quest the internal structure of black hole is an evolutionary problem. Near the horizons, the potential decreases exponentially as

$$
V_{T}^{I N}\left(r^{*}\right) \propto \exp \left(\kappa_{+} r^{*}\right), \quad r^{*} \rightarrow-\infty\left(r \rightarrow r_{+}\right)
$$

and

$$
V_{T}^{I N}\left(r^{*}\right) \propto \exp \left(-\kappa_{-} r^{*}\right), \quad r^{*} \rightarrow \infty\left(r \rightarrow r_{-}\right)
$$

It is useful to introduce the null coordinates $\left(v=r^{*}+t, u=r^{*}-t\right)$ to describe the inner structure of the charged black hole. In these coordinates the metric is given by $d s^{2}=f d v d u$. As is shown in Fig.2, the Cauchy horizon $r=r_{-}$consists of two branches (the right with $v=\infty$ and the left with $u=\infty)$. In order to find the energy density of the tachyon measured by a freely falling observer (FFO) with two-velocity $U^{\mu}\left(U^{\mu} U_{\mu}=-1\right)$, we have to consider the boundary conditions. Initially the tachyonic mode falls into the hole from the exterior region. After solving the equation near the horizons

$$
\left\{\frac{\partial^{2}}{\partial r^{* 2}}+\omega^{2}\right\} t_{\omega}^{I N}=0
$$

we have the ingoing wave near the event horizon $\left(r_{+}\right)$

$$
\left.t_{\omega}^{I N} e^{-i \omega t}\right|_{r_{+}}=T^{I N}(\omega) \exp \left\{-i \omega\left(t+r^{*}\right)\right\}
$$

On the other hand, the boundary condition near the Cauchy horizon is 


$$
\left.t_{\omega}^{I N} e^{-i \omega t}\right|_{r_{-}}=\exp \left\{-i \omega\left(t+r^{*}\right)\right\}+R^{I N}(\omega) \exp \left\{-i \omega\left(t-r^{*}\right)\right\}
$$

where the first term refers the ingoing mode into the left branch with $u=\infty$, while the second denotes the backscattered mode into the right branch with $v=\infty$. Here $T^{I N}(\omega)$ and $R^{I N}(\omega)$ are the transmission and reflection amplitudes for given mode $\omega$. We need the total tachyonic function $\left(\tilde{t}^{I N}\left(r^{*}, t\right)\right.$ in $\left.(20)\right)$ to obtain the energy density. This is given by the Fourier integral transfrom over the frequency $\omega$

$$
\tilde{t}^{I N}\left(r^{*}, t\right)=\int e^{-\sqrt{2} r} t_{\omega}^{I N} e^{-i \omega t} a(\omega) d \omega
$$

with the mode constant $a(\omega)$. Considering the boundary condition near the Cauchy horizon, this takes the form

$$
\left.\tilde{t}^{I N}(u, v)\right|_{r_{-}} \sim e^{-\sqrt{2} r}\left[t^{-}(u)+t^{+}(v)\right]
$$

From (12) and (20), the energy density measured by a FFO is dominated by [6]

$$
\rho=T_{\alpha \beta}^{t} U^{\alpha} U^{\beta} \sim\left|U^{\alpha} \tilde{t}^{I N},{ }_{\alpha}\right|^{2} \sim\left|U^{\alpha} t^{ \pm},{ }_{\alpha}\right|^{2}
$$

When a FFO1 crosses the left branch of the Cauchy horizon, one has

$$
U^{\alpha} t^{-},{ }_{\alpha} \propto t^{-\prime}(u) \exp \left(\frac{\kappa_{+} u}{2}\right)
$$

where the prime means the differentiation with respect to the given argument. In order to calculate $t^{-\prime}(u)$, we consider the deviation from the wave $\left(t_{\omega}^{I N}=\exp \left(-i \omega r^{*}\right)\right)$ treating $V_{T}^{I N}\left(r^{*}\right)$ in (43) as the infinitesimal perturbation. Following Ref.[6], we find $t^{-\prime}(u) \propto \exp \left(-\frac{\kappa_{+} u}{2}\right)$ as $u \rightarrow \infty$. Therefore this wave gives a finite energy density at the left Cauchy horizon. On the other hand, the energy density measured by a FFO2 who crosses the $\operatorname{right}(v \rightarrow \infty)$ horizon is proportional to the square of

$$
U^{\alpha} t^{+},_{\alpha} \propto t^{+\prime}(v) \exp \left(\frac{\kappa_{-} v}{2}\right)
$$

Substituting the form of $t^{+\prime}(v) \propto \exp \left(-\frac{\kappa_{+} v}{2}\right)$ into (51) together with $\kappa_{-}-\kappa_{+}>0$ leads to a divergent energy density on the right Cauchy horizon. The monochromatic tachyon waves 
with small amplitude and purely ingoing near the event horizon develop the infinite energy density near Cauchy horizon. This corresponds to the blueshift of tachyon. Further this means that the Cauchy horizon of the 2D charged black hole is unstable to the physical perturbations.

Now let us consider the same problem outside the black hole. Note that the last term $\left(\frac{Q^{2}}{8} e^{-4 \sqrt{2} r}\right)$ of $f$ in (14) decreases faster than the second $\left(e^{-2 \sqrt{2} r}\right)$, as $r$ increases. Outside the black hole, we then immediately recover the $2 \mathrm{D}$ dilaton black hole background as

$$
\bar{\Phi}=2 \sqrt{2} r, \quad \bar{F}_{t r} \approx 0, \quad \bar{T}=0, \quad \bar{G}_{\mu \nu} \approx\left(\begin{array}{cc}
-\tilde{f} & 0 \\
0 & \tilde{f}^{-1}
\end{array}\right)
$$

with

$$
\tilde{f}=1-e^{-2 \sqrt{2} r} .
$$

This corresponds to $Q=0$ case. The difference is that the position of event horizon is shifted from $r_{+}=-0.056$ (for $Q \neq 0$ ) to $r_{+}=0$. Actually $\tilde{V}_{T}^{\text {OUT }}$ in Fig. 3 ( potential for the $2 \mathrm{D}$ dilaton black hole) is approximately a copy of the right barrier $\left(V_{T}^{\text {OUT }}\right)$ in Fig.1 except the shift of $r_{+}$and scaling. These differences are not important to our considerations outside the black hole. For a simplicity, we use $\tilde{V}_{T}^{\text {OUT }}$ (instead of $V_{T}^{\text {OUT}}$ ) to investigate the exterior region. Here we briefly study how string modes propagate outside the 2D dilaton black hole [15]. Introducing $\tilde{r}^{*}=\tilde{g}(r)$, we have $\tilde{g}^{\prime} \tilde{f}=1$. Explicitly, one can find the form of $\tilde{g}(r)$ as

$$
\tilde{g}(r)=r+\frac{1}{2 \sqrt{2}} \ln \left(1-e^{-2 \sqrt{2} r}\right) .
$$

Note that $\tilde{r}^{*}$ ranges from $-\infty$ to $+\infty$, while $r$ ranges from the event horizon of the black hole $\left(r_{+}=0\right)$ to $+\infty$. We can visualize the $2 \mathrm{D}$ dilaton black hole as presenting a potential barrier (well) to the oncoming waves (for example, $t, h+\phi, h-\phi$ ). First let us discuss the tachyonic propagations outside the black hole. Assuming $t^{\text {OUT }}\left(r^{*}, t\right) \sim t_{\omega}^{\text {OUT }}\left(r^{*}\right) e^{-i \omega t}$, we find the equation for the tachyonic mode from (36)

$$
\left[\frac{\partial^{2}}{\partial r^{* 2}}+\omega^{2}-\tilde{V}_{T}^{O U T}\right] L^{O U T}=0,
$$


where $\tilde{V}_{T}^{O U T}$ is given by

$$
\tilde{V}_{T}^{\text {OUT }}=\tilde{f}\left(\sqrt{2} \tilde{f}^{\prime}-2(1-\tilde{f})\right)=2 e^{-2 \sqrt{2} r}\left(1-e^{-2 \sqrt{2} r}\right) .
$$

The scattering of tachyon by (56) was discussed in [15] and is shown in Fig.2. For gravitondilaton modes, (30) and (35) reduce to

$$
\begin{gathered}
\bar{\nabla}^{2}(h-\phi)=0, \\
\bar{\nabla}^{2}(h+\phi)+4 \sqrt{2} \partial_{r} \tilde{f}(h+\phi)=0 .
\end{gathered}
$$

Here we obtain two modes $(h-\phi)$ and $(h+\phi)$. Defining $H \equiv h-\phi$ and considering the trial solution of the form

$$
H^{O U T}\left(r^{*}, t\right) \sim H_{k}^{O U T}\left(r^{*}\right) e^{-i k t},
$$

we have the free field equation from (57)

$$
\left[\frac{\partial^{2}}{\partial r^{* 2}}+k^{2}\right] H_{k}^{O U T}\left(r^{*}\right)=0
$$

The other graviton-dilaton mode $(J \equiv h+\phi)$ is also given by the one-dimensional differential equation. Considering $J^{O U T}\left(r^{*}, t\right) \sim J_{k}^{O U T}\left(r^{*}\right) e^{-i k t}$, from (58) we obtain

$$
\left[\frac{d^{2}}{d r^{* 2}}+\left(k^{2}-V_{J}^{O U T}\right)\right] J_{k}^{O U T}=0
$$

where the potential well is given by

$$
V_{J}^{O U T}=-16 e^{-2 \sqrt{2} r}\left(1-e^{-2 \sqrt{2} r}\right)=\frac{-4}{\left(\cosh \sqrt{2} r^{*}\right)^{2}} .
$$

Outside 2D dilaton black hole, we find the well-known Schrödinger problem with the constant energy $\left(E=k^{2}\right)$ : a potential barrier (56) for the tachyonic mode, a potential well (62) for one graviton-dilaton mode $(h+\phi)$ and no potential for the other graviton-dilaton mode $(h-\phi)$. It is emphasized that the potential well for $J=h+\phi$ is obviously a new feature of $d=2$ black hole. From (61), one finds an exponentially growing mode with time 
$\left(e^{-i k t}, k=i \alpha\right)$. Naively this means that the event horizon is unstable. However, according to [16], $(h+\phi)$ and $(h-\phi)$ are not the physical degrees of freedom and nothing but gauge artifacts. Further it is explicitly shown that this exponentially growing mode with time can be removed by the coordinate transformation (just a translation) [17]. The tachyonic mode is also the physically propagating one outside the black hole. The stability should be based on the physical degree of freedom. With the potential barrier (56), one cannot find the bound state solutions which lead to the exponentially growing modes. Therefore the event (outer) horizon of $2 \mathrm{D}$ charged black hole is stable.

In summary, all string fields except the tachyon are non-propagating in the 2D charged black hole with double horizons. Only the tachyonic mode is a physically propagating one both inside and outside the black hole. The crucial problem has to do with the inner (Cauchy) horizon of the 2D charged black hole. Inside the black hole the radial coordinate $\left(r\right.$ or $\left.r^{*}\right)$ is timelike, whereas the time $(t)$ is spacelike. Hence to investigate the internal structure of black hole is an evolutionary problem. This horizon is believed to be unstable under tachyonic perturbation because it is a surface where the infalling matter got infinitely blueshifted [2-8]. On the other hand, outside the black hole we have the conventional scattering problem with the potntial barrier. The outer (event) horizon of $2 \mathrm{D}$ charged black hole is stable.

\section{ACKNOWLEDGMENTS}

This work was supported in part by the Basic Science Research Institute Program, Ministry of Education, Project NO. BSRI-95-2441 and by NONDIRECTED RESEARCH FUND, Korea Research Foundation, 1994. 


\section{REFERENCES}

[1] J. D. Brown, M. Henneaux and C. Teitelboim, Phys. Rev. D33, 319 (1986); B. B. Mann, A. Shiekh and L. Tarsov, Nucl. Phys. B 341, 134 (1990) ; E. Witten, Phys. Rev. D44, 314 (1991); G. Mandal, A. Sengupta and S. R. Wadia, Mod. Phys. Lett. A6, 1685 (1991); S.Elitzur, A. Forge and E. Rabinovici, Nucl. Phys. B 359, 581 (1991); S. P. deAlwis and J. Lykken, Phys. Lett. B269, 464 (1991);

[2] M. D. McGuigan, C. N. Nappi and S. A. Yost, Nucl. Phys. B 375, 421 (1992); O. Lechtenfeld and C. N. Nappi, Phys. Lett. B288, 72 (1992).

[3] R. Penrose, in Batelle Rencontres, edited by C. M. De Witt and J. A. Wheeler (Benjamin, New York, 1968).

[4] J. M. McNarama, Proc. Roy. Soc. Lon. A358, 499 (1978).

[5] Y. Gürsel, I. D. Novikov, V. D. Sandberg and A. A. Starobinsky, Phys. Rev. D19, 413 (1979).

[6] R. A. Matzer, N. Zamorano and V. D. Sandberg, Phys. Rev. D19, 2821 (1979).

[7] S. Chandrasekhar and J. Hartle, Proc. Roy. Soc. Lon. A384, 301 (1982).

[8] T. M. Helliwell and D. A. Konkowski, Phys. Rev. D47, 4322 (1993).

[9] E. Poisson and W. Israel, Phys. Rev. D41, 1796 (1990).

[10] A. Ori, Phys. Rev. Lett. 67, 789 (1991).

[11] V. Husain, Phys. Rev. D50, 2361 (1994).

[12] J. S. Chan and R. B. Mann, Phys. Rev. D50, 7376 (1994).

[13] C.G. Callan, D. Friedan, E,J. Martinec, and M.J. Perry, Nucl. Phys. B262, 593 (1985); T. Banks, Nucl. Phys. B361, 166 (1991).

[14] A. Peet, L. Susskind and L. Thorlacius, Phys. Rev. D48, 2415 (1993). 
[15] J.Y. Kim, H. W. Lee and Y. S. Myung, Phys. Lett. B328, 291 (1994); Phys. Rev. D50, 3942 (1994); Y. S. Myung, J.Y. Kim and C. Jue, Phys. Lett. B341, 273 (1995)

[16] Y. S. Myung, Phys. Lett. B341, 273 (1995).

[17] W. T. Kim, J. Lee and Y. J. Park, Phys. Lett. B347, 217 (1995). 


\section{FIGURES}

Fig.1: The graph of the effective potential of tachyon $\left(V_{T}(r)\right)$. This takes the doublehumped barrier well $\left(V_{T}^{I N}\right)$ inside the black hole, while it takes a simple potential barrier $\left(V_{T}^{\text {OUT }}\right)$ outside the black hole. The event horizon is at $r_{+}=-0.056$ and the Cauchy horizon is at $r_{-}=-0.679$.

Fig.2 : Conformal diagram of a portion of the 2D charged black hole space-time. Two observers are shown falling through $r=r_{+}$into the interior region and then through the Cauchy horizon at $r=r_{-}$. FFO1 (FFO2) cross the left (right) branches of $r=r_{-}$. An incident wave is scattered from the potential $\left(V_{T}^{\text {OUT }}\right)$, then proceeds into the interior region where further scattering by $V_{T}^{I N}$ occurs. The scattered wave will be rescattered into hole, to give a tail with a power-law (in time) decay. The energy density near the right branch due to this tail decays sufficiently slowly so that infinite energy densities are developed. These are measured by FFO2.

Fig.3 : The graph of the potential barrier of tachyon outside 2D dilaton black hole $\left(\tilde{V}_{T}^{\text {OUT }}\right)$. This is approximately a copy of right barrier $\left(V_{T}^{\text {OUT }}\right)$ in Fig.1. The apparent difference is due to a shifting from $r_{+}=-0.056(Q \neq 0)$ to $r_{+}=0$ and scaling. For a simplicity we use this potential to investigate the exterior region. The asymptotically flat region is located at $r=\infty$. 